\title{
Temperature characterization of dielectric permittivity and AC conductivity of nano copper oxide-doped polyaniline composite
}

\author{
L. N. Shubha** and P. Madhusudana Rao ${ }^{\dagger}$ \\ *Department of Electronics, St. Francis College for Women \\ Begumpet, Hyderabad 500011, Telangana, India \\ ${ }^{\dagger}$ Department of Physics, Jawaharlal Nehru Technological University Hyderabad \\ Kukatpally, Hyderabad 500085, Telangana, India \\ tshubhastfrancis@gmail.com \\ Received 10 January 2016; Accepted 6 May 2016; Published 28 June 2016
}

\begin{abstract}
The polyaniline/copper oxide $(\mathrm{PANI} / \mathrm{CuO})$ nanocomposite was prepared by mixing solutions of polyaniline and copper oxide nanoparticles in dimethyl sulfoxide (DMSO). The synthesized polymer nanocomposites were characterized by X-ray powder diffraction (XRD), scanning electron microscope (SEM) and UV-visible spectroscopy. The characteristic peaks in XRD and UVvisible spectra confirmed the presence of $\mathrm{CuO}$ in the polymer structure. SEM images indicated morphological changes in the composite matrix as compared to the pristine PANI. The DC conductivity measurements were performed using two-probe method for various temperatures. AC conductivity and dielectric response of the composites were investigated in the frequency range of $10^{2}-10^{6} \mathrm{~Hz}$ using LCR meter. Dielectric permittivity $\epsilon^{\prime}(w)$ and dielectric loss factor $\epsilon^{\prime \prime}(w)$ were investigated. It was observed that $\epsilon^{\prime}(w)$ and $\epsilon^{\prime \prime}(w)$ decrease with increase in frequency at all temperatures. At a particular frequency it is observed that both $\epsilon^{\prime}(w)$ and $\epsilon^{\prime \prime}(w)$ increase with increase in temperature. It was also observed that AC conductivity increased with increase in frequency and temperature.
\end{abstract}

Keywords: AC conductivity; dielectric permittivity; dielectric loss factor; polyaniline composite.

\section{Introduction}

The discovery of conducting polymers has opened up a promising field in material science and engineering. Among them, conducting polyaniline (PANI) is one of the most promising conducting polymers because of its unique electrical, optical and optoelectrical properties. In addition, it is easy to prepare and has excellent environmental stability. ${ }^{1-3}$

PANI can be used in electrochromic devices, light-emitting diodes, electrostatic discharge protection, etc. ${ }^{4-6}$ In recent years, one-dimensional PANI nanostructures, including nanowires, nanorods and nanotubes, have been studied. ${ }^{7-9}$ Nowadays, conducting PANI/metal oxide nanocomposites have also attracted attention. ${ }^{10}$ The properties of these nanocomposites are quite different from pristine PANI due to interfacial interactions between metal oxide nanoparticles and PANI macromolecules. ${ }^{11,12}$ The composite properties can be easily adjusted to the desired applications via the variation of particle size, shape and the distribution of nanoparticles. ${ }^{13,14}$ Consequently, PANI may have potential applications in electrical nanodevices. Polyaniline inorganic particle composites have been studied for applications as biosensors ${ }^{15}$ and in high-density storage devices. ${ }^{16}$ Successful preparation of conducting polymer as humidity sensor has been reported. ${ }^{17}$ There are reports of strong dielectric responses in selected conjugated polymeric structures. For example, a polymer composite consisting of the polymer metal oxide nanocomposites shows good dielectric response as well as good stability. ${ }^{18}$ Among those metal oxide nanoparticles, copper oxide $(\mathrm{CuO})$ nanoparticles are appealing because of their excellent physical and chemical properties. ${ }^{19} \mathrm{CuO}$ is frequently used as anode material for lithium ion batteries owing to its high charge capacity, safety and low cost. ${ }^{20}$ Therefore, the aim of the present work is to prepare PANI/CuO nanocomposites and to study their structure, dielectric properties and electrical properties. The present study will help in understanding the effect of temperature on electrical response of $\mathrm{PANI} / \mathrm{CuO}$ composite.

\section{Experimental}

\subsection{Materials}

Aniline, aniline hydrochloride powder, ammonium persulfate (APS: $\left.\left(\mathrm{NH}_{4}\right)_{2} \mathrm{~S}_{2} \mathrm{O}_{8}\right)$ and methanol were purchased from S. D. Fine Chem. Ltd., Mumbai. Aniline was distilled prior to use. All the solutions were made using double distilled water. Acetone, cupric nitrate and dimethyl sulfoxide (DMSO) were purchased from S.D. Fine Chem. Ltd., Mumbai.

This is an Open Access article published by World Scientific Publishing Company. It is distributed under the terms of the Creative Commons Attribution 4.0 (CC-BY) License. Further distribution of this work is permitted, provided the original work is properly cited. 


\subsection{Sample preparation}

\subsubsection{Copper oxide nanoparticle}

Nanostructure copper oxide was prepared by using $0.2 \mathrm{M}$ of cupric nitrate and dissolving it in $100 \mathrm{ml}$ of deionized water. This solution was stirred continuously for $1 / 2 \mathrm{~h}$. To this solution, $0.2 \mathrm{M} \mathrm{NaOH}$ was added dropwise till the $\mathrm{pH}$ of the solution was 10 . Black precipitate formed was centrifuged and washed with distilled water. This black powder was calcined at $350^{\circ} \mathrm{C}$.

\subsubsection{Polyaniline/copper oxide composite}

The PANI/CuO nanocomposite was prepared by solution mixing method. The complete preparation of polyaniline has been described in our previous publication. ${ }^{21}$ APS (0.2 M), used as an oxidant, was added slowly to aniline monomer $(0.2 \mathrm{M})$ to form polyaniline. The prepared polyaniline powder was dissolved in DMSO. Copper oxide was also dissolved in DMSO and sonicated. This solution was added to the prepared PANI solution. The resultant solution was stirred for $11 \mathrm{~h}$ at room temperature and filtered.

\subsection{Characterization}

UV-visible absorbance spectra were recorded on double beam UV-visible absorption spectrometer (Systronics 2201). The wavelength range of observation was from $200 \mathrm{~nm}$ to $1100 \mathrm{~nm}$. Wide angle X-ray powder diffraction (XRD) is used to obtain information about the structure, composition and state of polyaniline materials. X-ray diffractometer was used to characterize the samples with $\mathrm{Cu}-\mathrm{K} \alpha(1.5406 \AA)$ radiation using Philips PW 1530 diffractometer. The diffraction patterns were collected at the diffraction angle $2 \theta$ from $0^{\circ}$ to $80^{\circ}$ at a scanning rate and step size of 10 per min and $0.05^{\circ}$, respectively. The morphologies of $\mathrm{PANI} / \mathrm{CuO}$ composites were investigated by scanning electron microscope (SEM) on a Zeiss EVO 18(UK) instrument. DC conductivity measurements were made on compressed pellets of the powder using conventional two-probe technique at different temperatures. AC conductivity as well as dielectric measurements were investigated over the frequency range of $10^{2}-10^{6} \mathrm{~Hz}$ using Wein Kerr (UK) 41110 LCR meter.

\section{Results and Discussion}

\section{1. $U V$-visible spectra}

The UV-visible spectra of the synthesized PANI, $\mathrm{CuO}$ and $\mathrm{PANI} / \mathrm{CuO}$ nanocomposite in DMSO are shown in Fig. 1.

In the case of PANI, the peak occurs in the wave length range of $300-400 \mathrm{~nm}$ with the maximum at $335 \mathrm{~nm}$ and is attributable to $\pi-\pi^{*}$ transition of Benzenoid ring. The peak

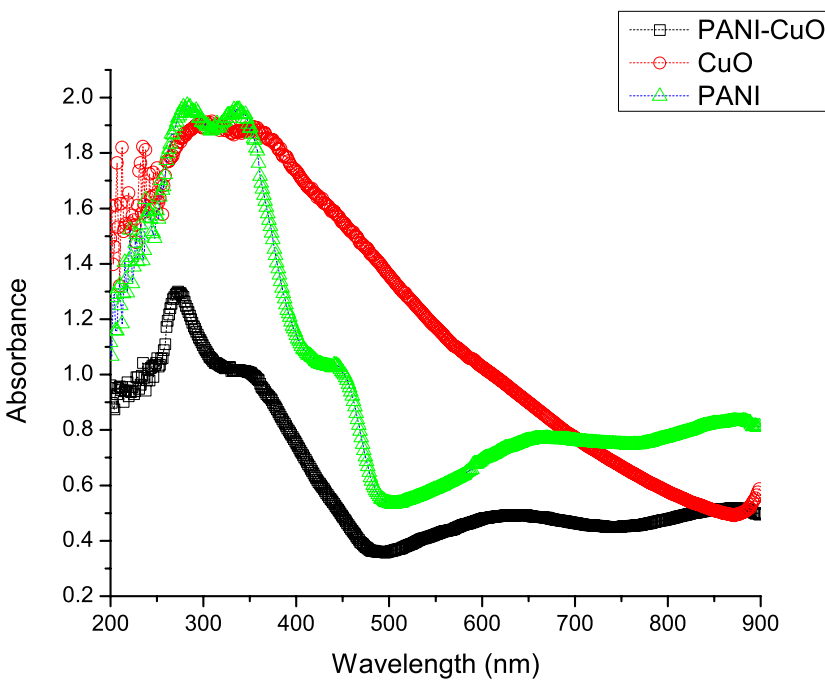

Fig. 1. UV-visible spectra for PANI, $\mathrm{CuO}$ and PANI/CuO composite.

at $445 \mathrm{~nm}$ is due to polaron $\pi^{*}$ transition and the broad band with absorption maximum at $620 \mathrm{~nm}$ corresponds to the transitions from localized benzene molecular orbital (MO) to a quinonoid $\mathrm{MO} .{ }^{22}$ In case of $\mathrm{CuO}$, a wide absorption peak is observed in the wavelength range of $305-355 \mathrm{~nm}$. In the case of PANI-CuO, the peaks are centered at 335, 445 and $660 \mathrm{~nm}$ and they show blue shift. This indicates the interaction between PANI and $\mathrm{CuO}$ nanocomposite. The magnitude of the bandgap is estimated by extrapolating absorption peak (linear portion) to zero absorption.

The bandgap is calculated by

$$
E_{g}=h c / \lambda,
$$

where $E_{g}$ is the energy of bandgap, $c$ is the speed of light, $h$ is Planck's constant and $\lambda$ is the wavelength of UV absorbed. It was found that energy bandgap of the composite increased with doping of $\mathrm{CuO}$ nanoparticles.

The energy bandgaps of PANI, $\mathrm{CuO}$ and their composite are given in Table 1.

\subsection{XRD studies}

Figure 2 presents the XRD spectra of a PANI, PANI-CuO nanocomposite and $\mathrm{CuO}$. The XRD profile of PANI does not show sharp peaks thus suggesting the amorphous nature of polymer samples. Some sharp peaks were observed between

$\begin{aligned} & \text { Table } \\
& \text { and PANI/CuO nanocomposite. }\end{aligned}$
\begin{tabular}{lcc}
\multicolumn{3}{l}{ 1. Bandgap of } \\
\hline S. No. & Sample & Bandgap (eV) \\
\hline 1. & PANI & 2.52 \\
2. & PANI/CuO & 2.81 \\
3. & $\mathrm{CuO}$ & 3.535 \\
\hline
\end{tabular}




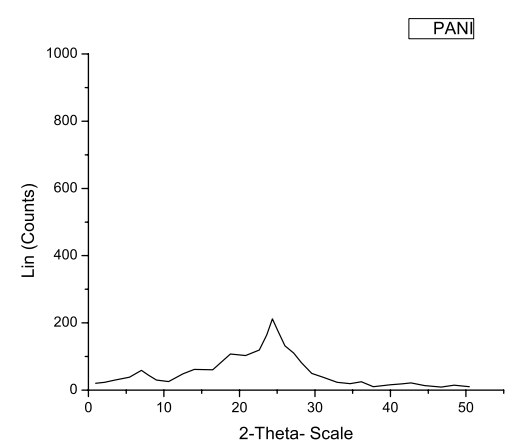

(a)

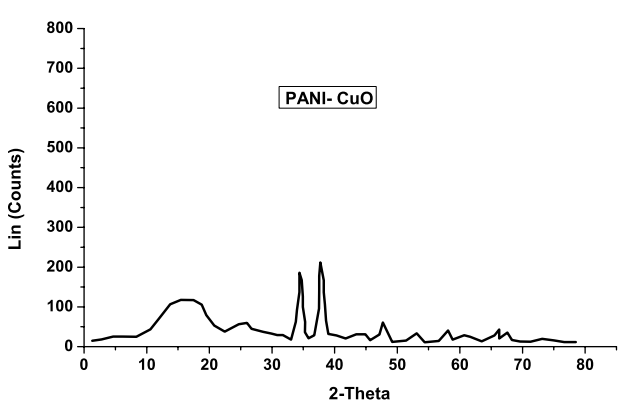

(b)

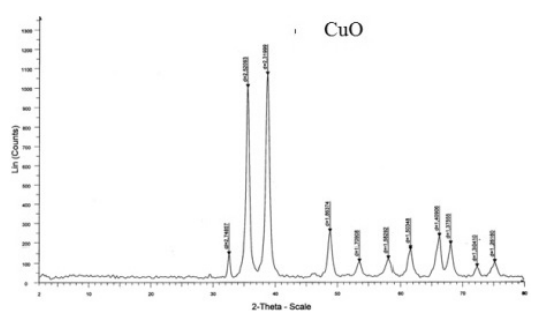

(c)

Fig. 2. XRD patterns of PANI, PANI/CuO composite and $\mathrm{CuO}$.

$20^{\circ}$ and $25^{\circ}$. This shows the semicrystalline nature of the samples. The main peaks of $\mathrm{CuO}$ nanoparticles are at $35.5^{\circ}$, $38.7^{\circ}$ and $48.7^{\circ}$ (JCPDS file no. 05-0661). These three peaks correspond to $(-111),(111)$ and $(-202)$ reflections, respectively. These peaks are also shown for PANI/CuO composite, which indicates the presence of $\mathrm{CuO}$ in PANI. Estimation of the crystallite size based on X-ray peaks broadening can be calculated using Debye-Scherrer equation. The Debye-Scherrer equation ${ }^{23}$ is given by

$$
t=\frac{0.9 \lambda}{\beta \cos \theta},
$$

where $t$ is the crystalline size of the particle, $\lambda$ is the $\mathrm{X}$-ray wavelength, $\beta$ is the full width at half maximum (FWHM) of the peak and $\theta$ is the Braggs angle. The calculated crystallite size of $\mathrm{CuO}$ is in the range of $50-60 \mathrm{~nm}$.

\subsection{Scanning electron microscopy}

Morphological study confirms the formation of PANI/CuO nanocomposite. Scanning electron micrographs of PANI/ $\mathrm{CuO}$ nanocomposites are shown in Fig. 3. The $\mathrm{CuO}$ nanoparticles exhibit granular structures. It is seen that the particles are agglomerated into irregular shape as a result of polymer growth on the surface of the nanoparticles.

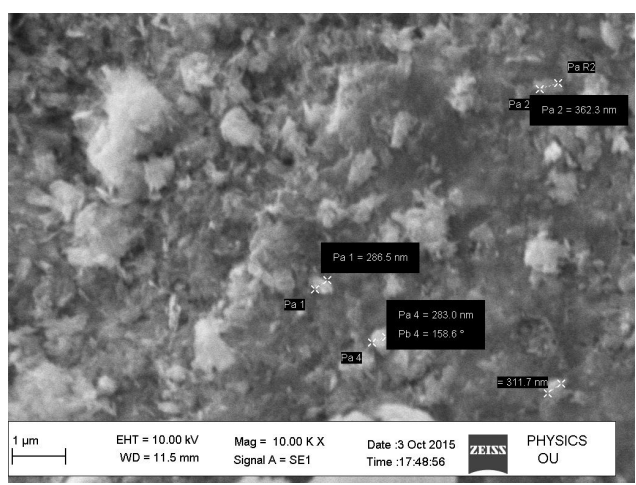

(a)

\subsection{Electrical investigation}

Electrical investigation is one of the most important characteristics of conducting polymers especially to explore their use in electrical devices. An attempt was made to measure DC conductivity at different temperatures. For electrical properties, the powder was made into pellets by uniaxial pressing.

\subsubsection{DC conductivity}

Room-temperature conductivity is measured on a pressed pellet using a two-probe conductivity measurement. It is represented in Table 2.

DC conductivity was calculated by

$$
\sigma_{\mathrm{dc}}=\frac{L}{R A},
$$

where $R$ is the resistance, $L$ is the thickness of the pellet and $A$ is the area of cross-section of the pellet. DC conductivity was also measured for varying temperature (from $25^{\circ} \mathrm{C}$ to $\left.100^{\circ} \mathrm{C}\right)$. It is observed that as the temperature is increased the resistivity decreases hence conductivity increases. ${ }^{24}$ So the polymer composite exhibits semiconductor behavior. Figure 4 is a plot of the DC conductivity as a function of temperature.

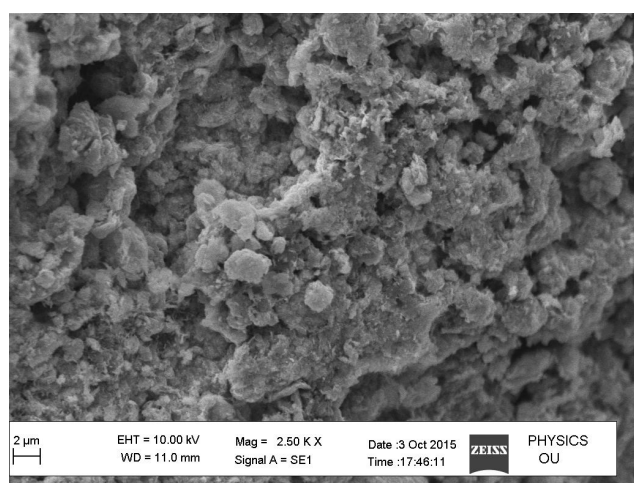

(b)

Fig. 3. SEM images of PANI/CuO composite (a) at a magnification of $10.00 \mathrm{kX}$ with marking and (b) at a magnification of $2.5 \mathrm{kX}$. 
Table 2. Conductivity measurement of PANI, $\mathrm{CuO}$ and polymer composite at $25^{\circ} \mathrm{C}$.

\begin{tabular}{lcc}
\hline S. No. & Sample & DC conductivity $(\mathrm{S} / \mathrm{cm})$ \\
\hline 1. & PANI & $4.17 \mathrm{E}-03$ \\
2. & PANI $/ \mathrm{CuO}$ & $1.21 \mathrm{E}-05$ \\
3. & $\mathrm{CuO}$ & $3.55 \mathrm{E}-06$ \\
\hline
\end{tabular}

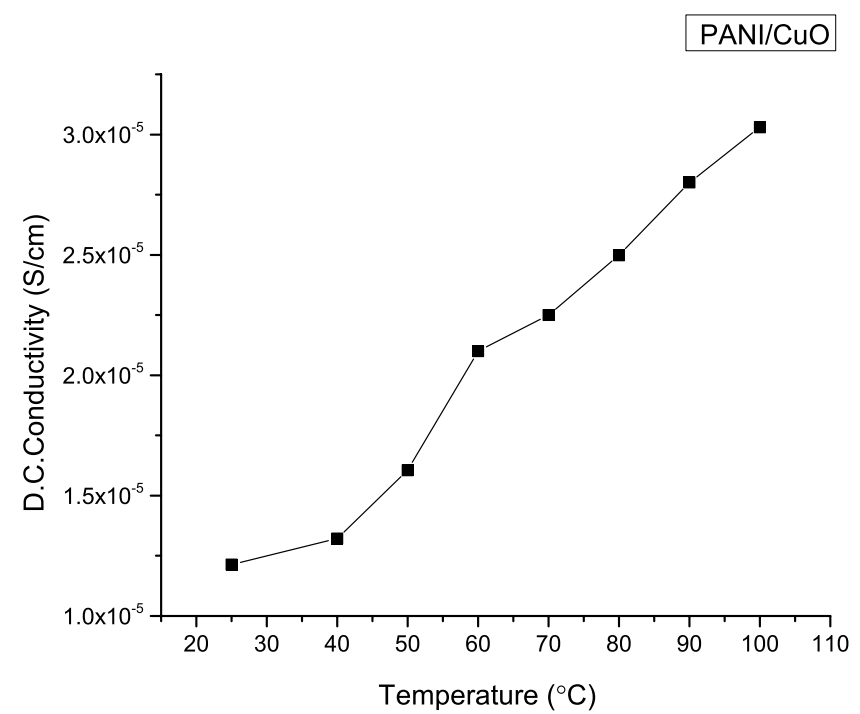

Fig. 4. DC conductivity of PANI/CuO composite as a function of temperature.

\subsubsection{Dielectric measurement}

For dielectric measurement, the capacitance $(C)$ and the dissipation factor $(D)$ for the sample were measured using an Wein Kerr 41110 (UK) LCR meter in the frequency range of $100 \mathrm{~Hz}-1 \mathrm{MHz}$. Figures 5 and 6 show the dielectric constant

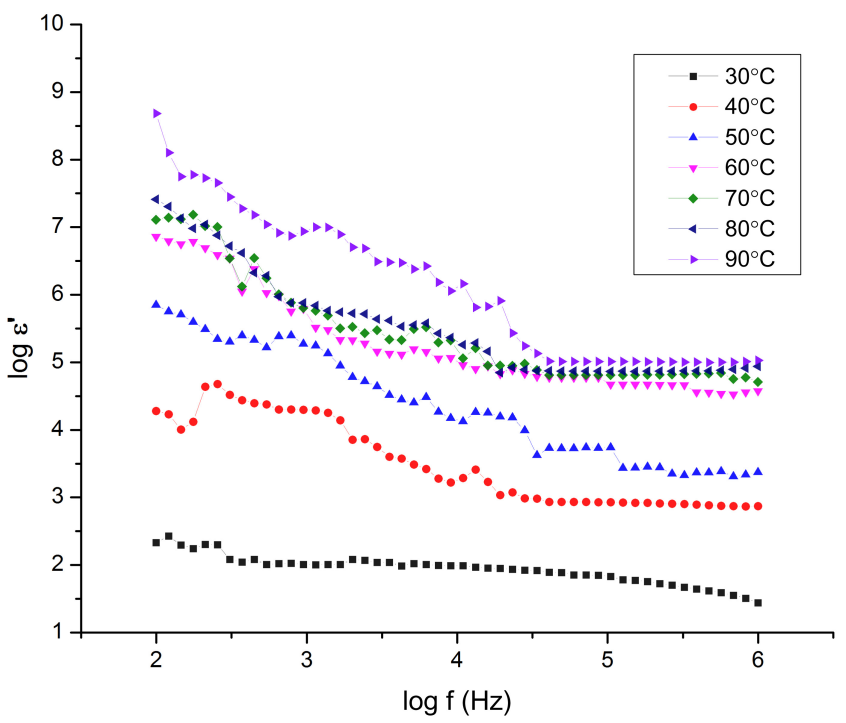

Fig. 5. Dielectric constant of $\mathrm{PANI} / \mathrm{CuO}$ as a function of frequency.

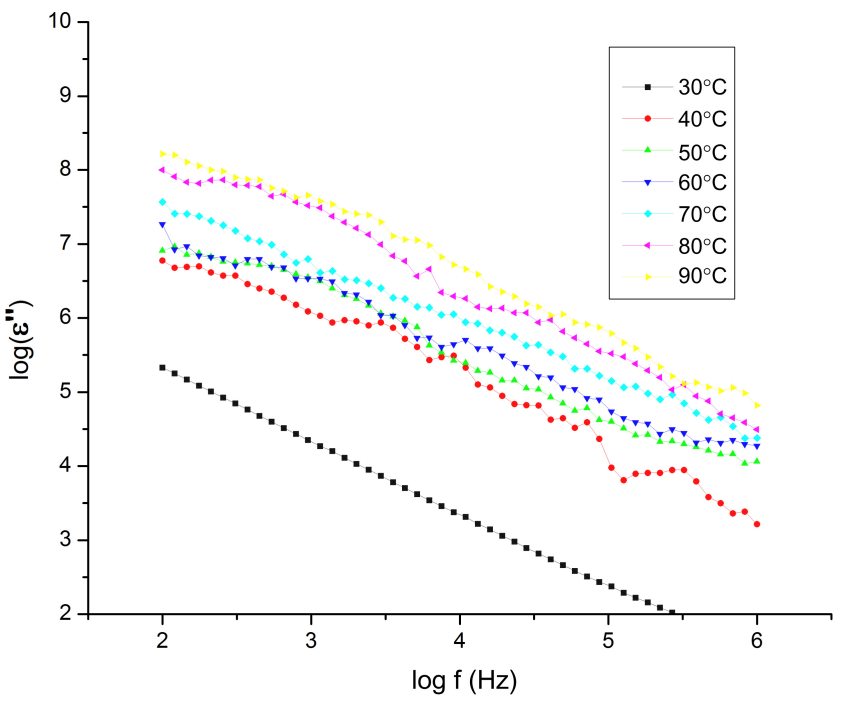

Fig. 6. Loss tangent of PANI/CuO as a function of frequency.

$\left(\epsilon^{\prime}(w)\right)$ and loss tangent $\left(\epsilon^{\prime \prime}(w)\right)$ of PANI/CuO nanocomposite versus frequency in the form of $\log -\log$ graph. At low frequencies, the sample exhibited high dielectric constant which decreased with increase in frequency. It is observed that the dielectric constant is independent of frequency beyond $100 \mathrm{kHz}$. The plots exhibit two linear portions of different slopes. This shows different polarization mechanisms in the low- and high-frequency ranges. In dielectric analysis, dielectric constant at high frequencies is associated with dipolar relaxation and at low frequencies, the dielectric constant is associated with interfacial polarization and DC conductivity. ${ }^{25}$ The increase in the dielectric constant at low frequencies reveals that system exhibits strong interfacial polymerization. Interfacial polarization occurs when there is an accumulation of charge between the two conductive and insulating regions within the material when electric field is applied. ${ }^{18}$ This interfacial polarization is particularly dominant at low frequencies and in conjugated polymers. At high frequencies, the variation in the field is very rapid for the dipoles to align themselves hence results in less dielectric constant. For PANI/CuO composite, the dielectric constant $\left(30^{\circ} \mathrm{C}\right)$ is 100.415 at $1 \mathrm{kHz}$ and it is around 63.095 at $100 \mathrm{kHz}$. From Fig. 6, it is observed that loss tangent of the sample decreases with increase in frequency.

The loss tangent is a combination of three distinct effects:

$$
\epsilon^{\prime \prime}(w)=\epsilon^{\prime \prime}(w)_{\mathrm{dc}}+\epsilon^{\prime \prime}(w)_{\mathrm{IP}}+\epsilon^{\prime \prime}(w)_{D},
$$

where $\epsilon^{\prime \prime}(w)_{\mathrm{dc}}$ is due to DC conduction, $\epsilon^{\prime \prime}(w)_{\mathrm{IP}}$ is due to interfacial polarization and $\epsilon^{\prime}(w)_{D}$ is due to oriental polarization (Debye type). The high value of loss tangent at low frequencies is mainly due to DC conduction and interfacial polarization. ${ }^{26}$ Figures 7 and 8 show the variations of dielectric constant and loss tangent as a function of temperature at various frequencies. It is observed that both $\epsilon^{\prime}(w)$ and $\epsilon^{\prime \prime}(w)$ increase with increase in temperature. This is because 


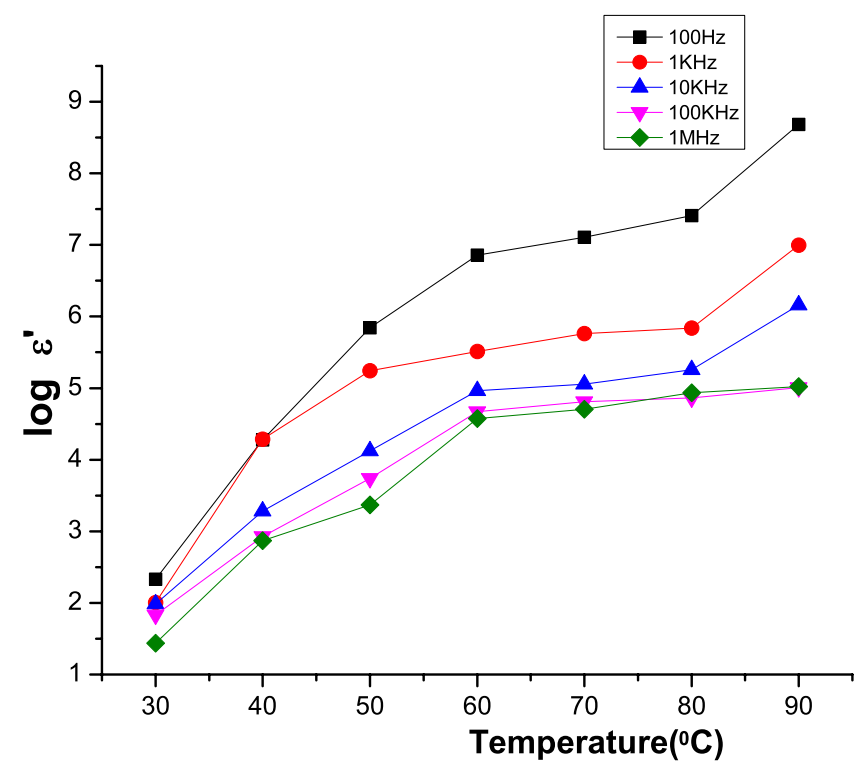

Fig. 7. Dielectric constant of $\mathrm{PANI} / \mathrm{CuO}$ as a function of temperature.

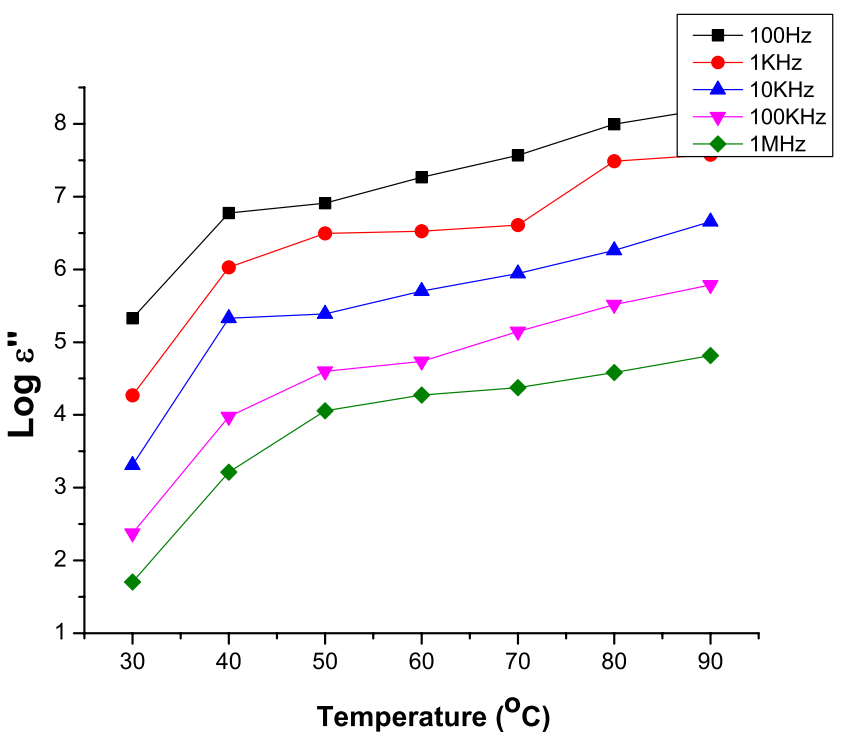

Fig. 8. Loss tangent of PANI/CuO as a function of temperature.

as the temperature of the sample increases, the dipoles comparatively become free and they respond to the applied electric field. Hence, polarization increases which leads to increase in dielectric constant. ${ }^{27,28}$

\subsubsection{AC conductivity}

The frequency-dependent conductivity of PANI/CuO composite is shown in Fig. 9. The AC conductivity $\left(\sigma_{\mathrm{ac}}\right)$ is obtained from using the relation:

$$
\sigma_{\mathrm{ac}}=\epsilon_{0} \epsilon^{\prime}(w) \omega D,
$$

where $\epsilon_{0}$ is the free space permittivity constant and $\omega=2 \pi f$ is the angular frequency.

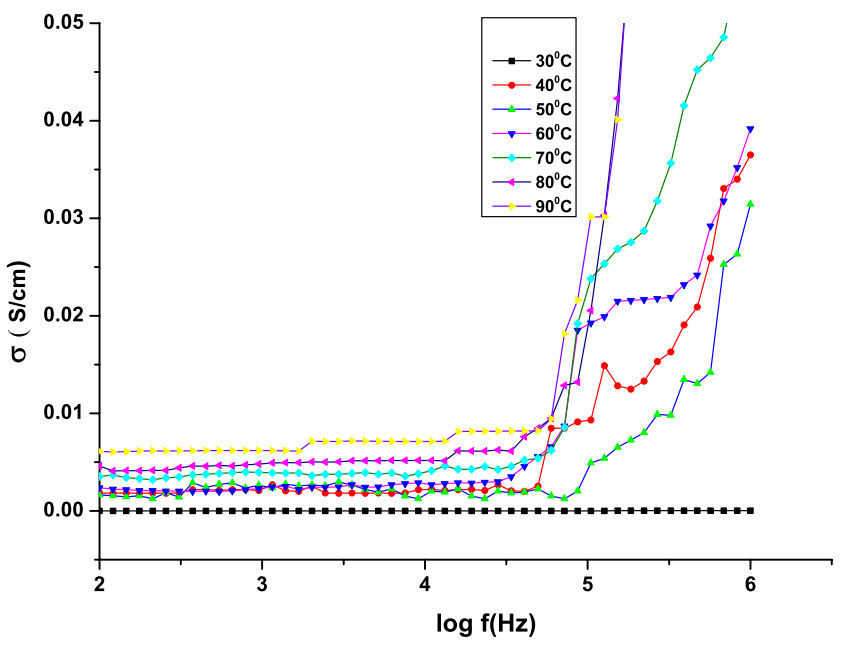

Fig. 9. AC conductivity of PANI/CuO as a function of frequency.

It is evident that the AC conductivity is frequency-dependent and is enhanced with increase in the frequency. It is also observed that $\mathrm{AC}$ conductivity remains constant at low frequencies and as frequency increases the conductivity increases exponentially. At all temperatures it is observed that above $100 \mathrm{kHz} \sigma_{\mathrm{ac}}$ increases exponentially. This frequency is called the critical frequency. AC conductivity obeys the power-law well above the critical frequency for the composite. The real part of AC conductivity can be expressed as

$$
\sigma_{\mathrm{ac}}=\sigma_{0}+A \omega^{n}
$$

where $\sigma_{0}$ denotes the DC conductivity, $A$ is a constant, $\omega$ is the angular frequency of applied electric field and $n \leq 1$ is a factorial exponent. ${ }^{29,30}$ The doping of conjugated polymers generates high conductivities by increasing the carrier concentration $n$. The polymer is oxidized by the acceptors (removal of electron), thereby producing a radical cation (hole) on the chain that does not delocalize completely, but is delocalized only over a few monomeric units deforming the polymeric structure. This radical cation with lattice distortion around the charge is called a polaron. This hole site moves through the polymer and it results in conductivity. Bipolarons are formed by removal of a second unpaired electron on a chain already having a negative or positive polaron. As these polarons and bipolarons are mobile they can move along the polymer chain. At higher frequencies, the conductivity increases because of the contribution of polarons which are moving along shorter distances in the polymer chain. The increasing $\sigma_{\mathrm{ac}}$ at higher frequencies is attributed to charge motion in the amorphous regions and this supports the presence of isolated polarons and bipolarons in this region. ${ }^{30}$ This indicates that there may be charge carriers which can be transported by hopping through the defect sites along the polymer chain. The frequency-dependent AC conductivity of $\mathrm{PANI} / \mathrm{CuO}$ increases as the temperature increases for a particular frequency. At all temperatures, it is observed that above $100 \mathrm{kHz} \sigma_{\mathrm{ac}}$ increases exponentially. The increase in 
the conductivity may be due to increased tunneling with rising temperature.

\subsection{Conclusions}

PANI and PANI/CuO composite were synthesized by solution mixing method. The incorporation of nano $\mathrm{CuO}$ in the PANI polymer matrix was confirmed by XRD, SEM and UV-visible spectra. The XRD pattern indicated the crystalline phase of $\mathrm{CuO}$. PANI/CuO composite samples have been associated with decrease in DC conductivity due to doping of $\mathrm{CuO}$ in PANI. It is observed that as the temperature is increased the conductivity increases. Hence, the polymer composite exhibits semiconductor behavior. AC conductivity obeys the power-law well above the critical frequency for the composite. AC conductivity increased exponentially above critical frequency for all temperature variations. Polymer composite exhibited increased values of both dielectric constant and loss tangent with decrease in frequency and increase in the temperature. It has been studied that variation in temperature on $\mathrm{PANI} / \mathrm{CuO}$ composite showed a prominent effect on conductivity and dielectric properties.

\section{Acknowledgments}

This work is supported by SERO-UGC (India), No. MRP5883/15, under the Minor Research Project Scheme. We thank Prof. Suresh Babu, Head of the Department of Physics, Osmania University for providing the test facility. Our thanks to Dr. B. Sreedhar of Inorganic and Organic Lab, IICT, Hyderabad for valuable suggestions.

\section{References}

${ }^{1}$ S. Xing, C. Zhao, S. Jing and Z. Wang, Morphology and conductivity of polyaniline nanofibers prepared by 'seeding' polymerization, Polymer 47, 2305 (2006).

${ }^{2}$ L. Yang, S. Wang, J. Mao, J. Deng, Q. Gao, Y. Tang and O. G. Schmidt, Hierarchical $\mathrm{MoS}_{2}$ /polyaniline nanowires with excellent electrochemical performance for lithium-ion batteries, $A d v$. Mater. 25, 1180 (2013).

${ }^{3}$ I. Venditti, I. Fratoddi, M. V. Russo and A. Bearzotti, A nanostructured composite based on polyaniline and gold nanoparticles: Synthesis and gas sensing properties, Nanotechnology 24, 155503 (2013).

${ }^{4}$ N. Gandhi, K. Singh, A. Ohlan, D. Singh and S. Dhawan, Thermal, dielectric and microwave absorption properties of polyaniline- $\mathrm{CoFe}_{2} \mathrm{O}_{4}$ nanocomposites, Compos. Sci. Technol. 71, 1754 (2011).

${ }^{5}$ S. Tan, J. Zhai, M. Wan, Q. Meng, Y. Li, L. Jiang and D. Zhu, Influence of small molecules in conducting polyaniline on the photovoltaic properties ofsolid-state dye-sensitized solar cells, J. Phys. Chem. B 108, 18693 (2004).

${ }^{6}$ G. B. Blanchet, C. R. Fincher and F. Gao, Polyaniline nanotube composites: A high-resolution printable conductor, Appl. Phys. Lett. 82, 1290 (2003).
${ }^{7}$ J. Huang and R. B. Kaner, Nanofiber formation in the chemical polymerization of aniline: A mechanistic study, Angew. Chem. 116, 5941 (2004).

${ }^{8}$ R. C. Y. King and F. Roussel, Morphological and electrical characteristics of polyaniline nanofibers, Synth. Met. 153, 337 (2005).

${ }^{9}$ A. N. Aleshin, H. J. Lee, Y. W. Park and K. Akagi, One-dimensional transport in polymer nanofibers, Phys. Rev. Lett. 93, 196601 (2004).

${ }^{10} \mathrm{C}$. Bian, Y. Yu and G. Xue, Synthesis of conducting polyaniline/ $\mathrm{TiO}_{2}$ composite nanofibres by one-step in situ polymerization method, J. Appl. Polym. Sci. 104, 21 (2007).

${ }^{11} \mathrm{~J}$. Alam, U. Riaz and S. Ahmad, Effect of ferrofluid concentration on electrical and magnetic properties of the $\mathrm{Fe}_{3} \mathrm{O}_{4} / \mathrm{PANI}$ nanocomposites, J. Magn. Magn. Mater. 314, 93 (2007).

${ }^{12}$ S. I. A. Razak, A. L. Ahmad and S. H. S. Zein, Polymerisation of protonic polyaniline/multi-walled carbon nanotubes-manganese dioxide nanocomposites, J. Phys. Sci. 20, 27 (2009).

${ }^{13} \mathrm{D}$. Saini and T. Basu, Synthesis and characterization of nanocomposites based on polyaniline-gold/graphene nanosheets, Appl. Nanosci. 2, 467 (2012).

${ }^{14}$ A. Dey, A. Kaushik, S. K. Arya and S. Bhansali, Mediator free highly sensitive polyaniline-gold hybrid nanocomposite based immunosensor for prostate-specific antigen (PSA) detection, J. Mater. Chem. 22, 14763 (2012).

${ }^{15} \mathrm{~S}$. Sedaghat, Synthesis and characterization of new biocompatible copolymer: Chitosan-graft-polyaniline, Int. Nano Lett. 499 (2014).

${ }^{16}$ A. Arslan and E. Hür, Supercapacitor applications of polyaniline and poly ( $n$-methylaniline) coated pencil graphite electrode, Int. J. Electrochem. Sci. 7, 12558 (2012).

${ }^{17}$ N. Parvatikar, S. Jain, S. Khasim, M. Revansiddappa, S. Bhoraskar and M. A. Prasad, Electrical and humidity sensing properties of polyaniline/ $\mathrm{WO}_{3}$ composites, Sens. Actuators B, Chem. 114, 599 (2006).

${ }^{18}$ N. Pinto, A. Acosta, G. Sinha and F. Aliev, Dielectric permittivity study on weakly doped conducting polymers based on polyaniline and its derivatives, Synth. Met. 113, 77 (2000).

${ }^{19}$ A. Liu, L. H. Bac, J.-S. Kim, B.-K. Kim and J.-C. Kim, Synthesis and characterization of conducting polyaniline-copper composites, J. Nanosci. Nanotechnol. 13, 7728 (2013).

${ }^{20}$ H. E. Z. Taghipour and M. Tanzifi, Modification of polyaniline/ polystyrene and polyaniline/metal oxide structure by surfactant, IJE TRANSACTION B. 27, 227 (2014).

${ }^{21}$ L. N. Shubha and P. Madhusudana Rao, In situ chemical synthesis and electrical properties of polyaniline/titanium dioxide nanocomposites, Int. J. Sci. Eng. Res. 6, 855 (2015).

${ }^{22} \mathrm{D}$. Deepshikha and T. Basu, Tuning of chemical switching properties of nanostructured conducting polyaniline using structure directing agents, Sci. J. Biotechnol. 175, 3 (2011).

${ }^{23}$ J. Stejskal and R. Gilbert, Polyaniline, preparation of a conducting polymer (IUPAC technical report), Pure Appl. Chem. 74, 857 (2002).

${ }^{24}$ E. Vitoratos, S. Sakkopoulos, E. Dalas, P. Malkaj and C. Anestis, DC conductivity and thermal aging of conducting zeolite/polyaniline and zeolite/polypyrrole blends, Curr. Appl. Phys. 7, 578 (2007).

${ }^{25}$ Y. Ravikiran, M. Lagare, M. Sairam, N. Mallikarjuna, B. Sreedhar, S. Manohar, A. MacDiarmid and T. Aminabhavi, Synthesis, characterization and low frequency $\{\mathrm{AC}\}$ conduction of polyaniline/ niobium pentoxide composites, Synth. Met. 156, 1139 (2006). 
${ }^{26}$ B. G. Soares, M. E. Leyva, G. M. Barra and D. Khastgir, Dielectric behavior of polyaniline synthesized by different techniques, Eur. Polym. J. 42, 676 (2006).

${ }^{27} \mathrm{X}$. Yan and T. Goodson, High dielectric hyperbranched polyaniline materials, J. Phys. Chem. B 110, 14667 (2006).

${ }^{28}$ N. N. Mallikarjuna, S. K. Manohar, P. V. Kulkarni, A. Venkataraman and T. M. Aminabhavi, Novel high dielectric constant nanocomposites of polyaniline dispersed with $\nu$ - $\mathrm{Fe}_{2} \mathrm{O}_{3}$ nanoparticles, J. Appl. Polym. Sci. 97, 1868 (2005).
${ }^{29}$ V. J. Babu, V. P. Kumar, G. Subha, V. Kumari, T. Natarajan, A. S. Nair and S. Ramakrishna, AC conductivity studies on PMMAPANI (HCL) nanocomposite fibers produced by electrospinning, J. Engineered Fiber. Fabr. 6, 54 (2011).

${ }^{30}$ A. B. Afzal, M. J. Akhtar, M. Nadeem, M. Ahmad, M. M. Hassan, T. Yasin and M. Mehmood, Structural and electrical properties of polyaniline/silver nanocomposites, J. Phys. D, Appl. Phys. 42 , 015411 (2009). 